\title{
SPONTANEOUS RESOLUTION OF PSEUDOANEURYSM OF AN ILIAC ARTERY BRANCH IN A MULTIPLE TRAUMA PATIENT WITH PELVIC FRACTURE: CLINICAL CASE
}

\author{
Cristina Maria Varino Sousa ${ }^{1}$, Luís Filipe Pires Silva', Maria Elisa Rodrigues ${ }^{1}$, António Félix', Bruno Alpoim ${ }^{1}$, Pedro Marques ${ }^{1}$, Joana \\ Alexandra Gonçalves Oliveira ${ }^{2}$, Carlos Alves ${ }^{3}$, Maieiro Costa $^{3}$, António Rodrigues ${ }^{4}$
}

\begin{abstract}
In patients who have been the victims of high-energy trauma, severe pelvic injury should always be suspected. Most of these fractures are stable and respond well to conservative treatment. Pelvic fractures constitute $3 \%$ of all skeletal fractures and are associated with high-energy trauma. They are potentially serious injuries with significant mortality and large numbers of associated lesions. There are fundamentally three sources of bleeding in pelvic fractures: arterial, venous and through the bone ends of the fracture. Arterial bleeding is more associated with hemodynamic
\end{abstract}

\section{INTRODUCTION}

Severe pelvic injuries have considerable incidence at emergency services in Portugal, particularly among the victims of high-energy trauma. They are more common among men, traffic accident victims and individuals in their third decade of life. Pelvic fractures indicate that high energy was transferred, with severe trauma $^{(1)}$. It has been estimated that, for the pelvic ring to be ruptured, a frontal collision at a speed of at least $48 \mathrm{~km} / \mathrm{h}$ or a lateral collision at $24 \mathrm{~km} / \mathrm{h}$ would be necessary. This great dissipation of energy is responsible for the associated lesions that are frequently present ${ }^{(2)}$.

Significant advances have been achieved over recent decades. On the one hand, there has been progress within surgery, with the use of external fixators to reduce unstable pelvic fractures, thereby making it possible to instability. In such cases, both early external fixation of the pelvic fracture and angiography with selective embolization of the bleeding vessels are effective methods for achieving hemostasis. Aneurysms of iliac artery branches are rare and are mostly pseudoaneurysm relating to the traumatic event. The natural history of pseudoaneurysms is unknown because of their rarity, but if they rupture, the mortality rate is high. We report a case of spontaneous thrombosis of a pseudoaneurysm of a branch of the right iliac artery.

Keywords - Hip Fractures; False Aneurysm; Iliac Artery

restore mobility to the patient. On the other hand, diffusion of advanced trauma life support (ATLS) theories has contributed towards diminishing mortality among patients with pelvic fractures ${ }^{(3)}$.

The prognosis for pelvic fracture victims seems mostly to be related to the associated lesions, given that such fractures are often mild and do not give rise to great hemorrhage. However, in some cases, voluminous retroperitoneal hemorrhage may occur, which may sometimes be lethal ${ }^{(2)}$.

At the beginning of the twentieth century, mortality among multiple trauma victims with pelvic fractures reached $80 \%$, especially due to retroperitoneal hemorrhage. Both the fracture surface and the rich vascular network present in this region are important sources of bleeding. Even with the concept of blood volume

\footnotetext{
1 - Complementary Orthopedics and Traumatology Clinician at Local Healthcare Unit, Alto Minho, SA, Portugal.

2 - Complementary Neurosurgery Clinician at Hospital de São João, EPE

3 - Specialist Orthopedics and Traumatology Clinician at Local Healthcare Unit, Alto Minho, SA, Portugal.

4 - Specialist Physician and Director of Orthopedics and Traumatology Service, Local Healthcare Unit, Alto Minho, SA, Portugal.
}

Work performed at the Orthopedics and Traumatology Service, Local Healthcare Unit, Hospital de Santa, Alto Minho, SA, Portugal.

Correspondence: Rua da Praia 204m - $3^{\circ}$ direito - Aguçadoura - 4495-031 - Póvoa de Varzim, Portugal. E-mail: crisvsousa@yahoo.com

Work received for publication: December 21, 2009; accepted for publication: July 26, 2010. 
replacement and progress in support measures, mortality persisted at close to $30 \%$ in the 1960 s. Early external fixation of pelvic fractures, in association with percutaneous angiography with selective embolization of the vessels with active bleeding, was responsible for changing the prognosis for these patients. Flint et $\mathrm{al}^{(4)}$ reported that the mortality rate was five times lower when angiography and embolization were used. Riemer et $\mathrm{al}^{(5)}$ observed that there was a significant decrease in mortality if early external fixation was implemented for complex pelvic fractures. Currently, the mortality rate ranges from 7 to $23 \%$. Among multiple trauma victi$\mathrm{ms}$, hemorrhage is the main cause of death $(39 \%$ of the cases), followed by associated cranioencephalic trauma $(35 \%)$ and sepsis with multiple organ failure $(25 \%)$.

At our service, the protocol for dealing with multiple trauma victims with pelvic fractures includes an initial assessment in accordance with the ATLS proposals. After the initial radiological investigation, abdominal and pelvic assessments of hemodynamically stable patients are made using computed tomography.

Vascular lesions of the iliac arteries are more common in cases of penetrating wounds ${ }^{(6)}$. However, closed pelvic trauma may lead to lesions in several branches of these arteries. Such lesions may result in fatal hemorrhage and should always be taken into consideration in cases of occult major hemorrhage. The vascular surgeon should be promptly consulted if arterial lesions are suspected or present. In cases of severe arterial hemorrhage, the initial treatment includes direct compression of the wound and blood volume resuscitation.

Lesions of the iliac artery caused by pelvic fractures are not common. Hemorrhage in cases of pelvic fracture, resulting from lesions of the internal iliac artery and its branches, is more common in cases of posterior pelvic fracture. Fractures due to lateral compression more often cause hemorrhage of the pudendal and obturator vessels ${ }^{(7)}$.

Vessels with lesions may be extremely difficult to identify, and attempting to do so on the operating table may result in abundant hemorrhage and fatal shock ${ }^{(6)}$. Arteriography is indicated for hemodynamically stable patients, both for diagnosing and for treating hemorrhage by means of embolization.

The therapeutic options have evolved over recent years and go from traditional surgery to approaches that are less invasive, and they include radiological procedures such as echo-guided compression, echo-guided percutaneous thrombin injection and endovascular procedures (embolization and placement of endoprostheses) ${ }^{(8)}$.
Endovascular approaches are the gold standard for treating deep arterial hemorrhage ${ }^{(9,10)}$.

Intervention radiology enables selective embolization of arteries that external fixation is unable to plug. During angiography, signs of macrovascular lesions that would be sources of extravasation of contrast medium (false aneurysm), arteries with wall irregularities, arteries that are unfilled downstream, or stagnation of contrast medium in veins, are sought ${ }^{(11)}$.

The time at which pseudoaneurysm is presented is variable and can be at any time from the original injury until years later ${ }^{(12)}$.

Damage to the arterial wall and extravasation of blood to the periarterial fascial plane may result in a pseudoaneurysm ${ }^{(13)}$. Thus, pseudoaneurysms result from transmural rupture of the arterial wall, extravasation of blood and formation of a hematoma that remains in communication with the arterial lumen. Unlike true aneurysms, pseudoaneurysms lack the three layers of the vessel wall (intima, media and adventitia) and are contained by perivascular tissue. Coagulation occurs on the periphery of the hematoma, which on ultrasound examination appears to be hyperechoic, while the center remains anechoic. During the intra-arterial high pressure of the systole, the blood flow is anterograde towards the pseudoaneurysm; during the diastole, the flow direction is retrograde. This phenomenon causes swirling, which is the usual flow pattern seen on Doppler images ${ }^{(14)}$.

Pseudoaneurysms of branches of the iliac arteries account for a tiny percentage of all false aneurysms ${ }^{(15)}$. They are usually asymptomatic, except when they rupture ${ }^{(16)}$. Individuals with this disease usually have a previous history of trauma, and this disease can also be diagnosed as a surgical finding ${ }^{(12)}$.

Pseudoaneurysms may undergo spontaneous thrombosis, or may evolve with development of complications such as infections, development of local compression over the neurovascular structures, or rupture.

Dilution coagulopathy may also be a reason for concern with regard to patients who receive large quantities of fluids. Thus, two to three units of frozen fresh plasma and seven to eight units of platelets should be prescribed for every five liters of volume replacement ${ }^{(1)}$.

\section{CLINICAL CASE}

The patient was a 22-year-old man who had been the victim of a traffic accident (head-on collision between a two-wheel vehicle that he was controlling and 
a four-wheel vehicle). Under observation in the emergency service on admission, he was hemodynamically stable and presented Glasgow 15 . He presented chest trauma, with bruising of the lungs, trauma to the pelvis with fracturing in the left wing of the sacrum, fracturing of the right ileopubic branch and fracturing of the right acetabulum without displacement. Computed tomography on the abdomen and pelvis showed that the pelvis was fractured on the left side, with a fracture of the sacrum wing and the obturator ring, and on the right side, with a fracture of the anterior pillar of the acetabulum and ischiopubic branch. None of these fractures presented any significant displacement. There were also fractures of the L2, L3 and L4 right transverse apophyses, without signs of fracturing of the vertebral bodies or posterior elements of the lumbar spine (Figure 1). There were no other apparent abdominal or pelvic lesions. The patient was administered fluid therapy and a transfusion of red blood cell concentrate, and remained clinically stable. It was decided to use conservative treatment for the pelvic fractures, consisting of resting in bed and skin traction on the right leg.

Eight days later, the patient started to complain of increased discomfort in the right lumbar region, with palpable tumefaction, accompanied by a fall in hemoglobin levels and hemodynamic instability (hypotension and tachycardia). Transfusional support was needed.

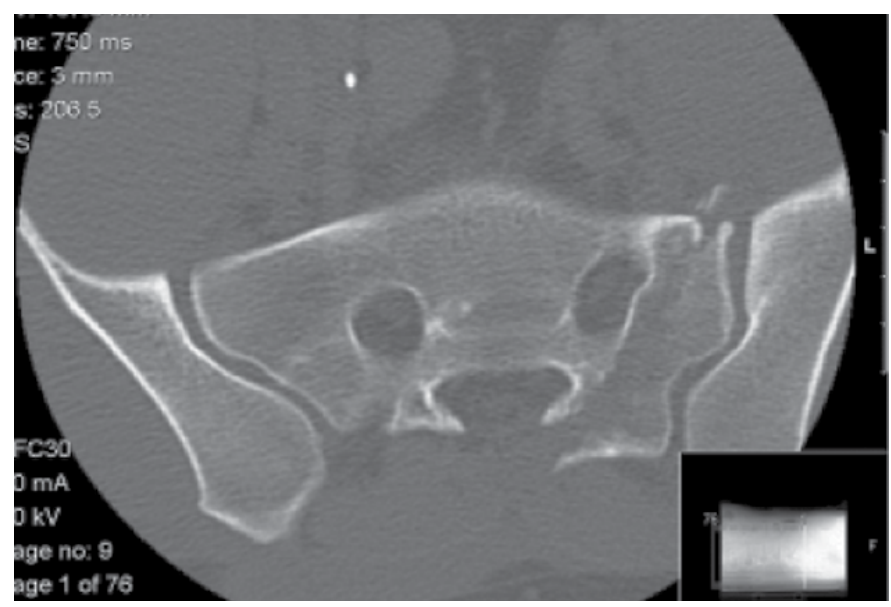

Figure 1 - Radiograph of the pelvis and axial computed tomography - images of pelvic fractures.

Computed tomography scans over the chest, abdomen and pelvis were produced again on an emergency basis, and these showed that in association with the fracture of the left half of the sacrum, there was a deep and voluminous subcutaneous hematoma in the lumbar and sacral regions that had increased greatly in relation to the previous examination. This was believed to be the

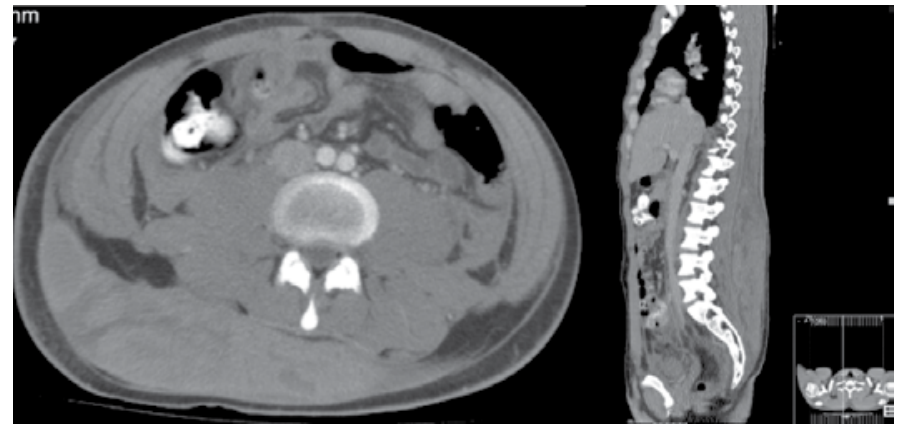

Figure 2 - Axial computed tomography showing increased density and edema in the dorsal-lumbar tissue layers and the right buttock region, with a voluminous hematoma.

cause of the decrease in the patient's hemoglobin levels (Figure 2). At this point, the patient was transferred to Hospital de São João, for vascular surgical care. Computed angiotomography was performed on an emergency basis, and this confirmed that a voluminous hematoma was present in the dorsal-lumbar-sacral region. An image of a sac measuring $37 \mathrm{~mm}$ with contrast inside (caliber of $21 \mathrm{~mm}$ ), which suggested the presence of a pseudoaneurysm in a branch of the right internal iliac artery (Figure 3).

Embolization of the false aneurysm was scheduled three days later, but this attempt did not succeed because the lesion was only poorly visible. One week later, the patient underwent arteriography, which did not show any evidence of the false aneurysms that had previously been encountered, thus showing spontaneous resolution (Figure 4).

During the hospital stay, clinical stability was maintained, without any evidence of worsening of the lumbar hematoma or signs of peritoneal irritation, deep vein thrombosis or long-limb ischemia. There was hemodynamic stability and the hemoglobin level remained unchanged ( $\mathrm{Hb} 12 \mathrm{~g} / \mathrm{dl})$.

\section{DISCUSSION}

In our sample, most of the pelvic fractures were stable and did not require specific treatment. However, a considerable proportion of such patients need specific attention for treatment of pelvic fractures; if this is not undertaken, increased mortality and complications should be expected. Even in large trauma centers, more than $60 \%$ of pelvic fractures are stable and do not require surgical stabilization. Among those that do need to be stabilized, most undergo external fixation and only $40 \%$ undergo internal fixation ${ }^{(3)}$.

Pseudoaneurysms of branches of the iliac artery are rare and are related to trauma, pelvic fractures or 

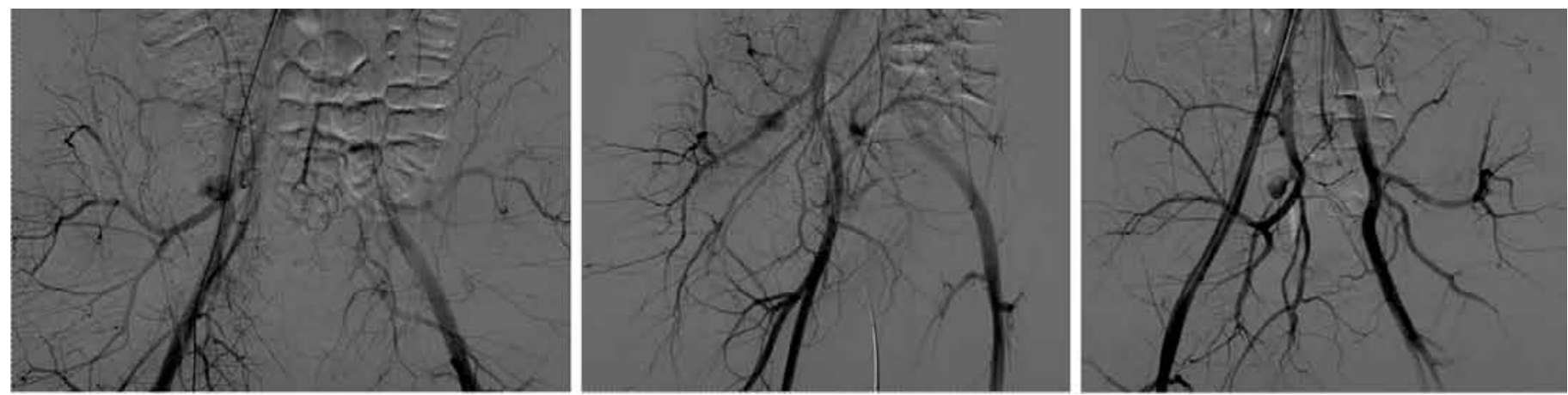

Figure 3 - Images of the first angiography performed. Adjacent to the internal iliac artery, an image of a sac measuring $37 \mathrm{~mm}$, with contrast medium inside it (caliber of $21 \mathrm{~mm}$ ), thus suggesting the presence of a pseudoaneurysm in a branch of the right internal iliac artery.

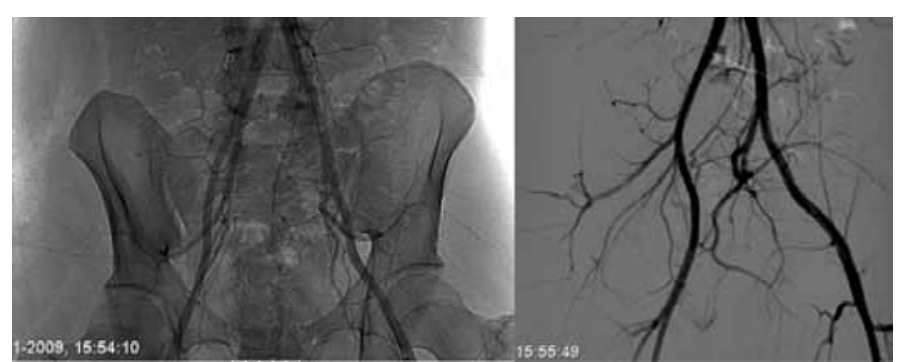

Figure 4 - Images from the last angiography performed, in which the occlusion of the previously diagnosed pseudoaneurysm can be seen.

iatrogenic lesions ${ }^{(17)}$. They are difficult to diagnose because physical examination is almost impossible. They tend to be asymptomatic, unless rupture occurs, and this may occur several days or months after the initial trauma ${ }^{(14)}$.

This case is of particular interest because the images obtained initially showed a small aneurysm of a branch of the internal iliac. The second angiography showed that the false aneurysm had decreased in size and the

\section{REFERENCES}

1. Bodden J. Treatment options in the hemodynamically unstable patient with a pelvic fracture. Orthop Nurs. 2009;28(3):109-14.

2. Parreira JG, Haddad L, Rasslan S. Lesões abdominais nos traumatizados com fraturas de bacia. Rev Col Bras Cir. 2002;29(3):153-60,

3. Chueire AG, Carvalho Filho G, Santos AF, Pockel KP. Fraturas do anel pélvico: estudo epidemiológico. Acta Ortop Bras. 2004;12(1):5-11.

4. Flint L, Babikian G, Anders M, Rodriguez J, Steinberg S. Definitive control of mortality from severe pelvic fracture. Ann Surg. 1990;211(6):703-6.

5. Riemer BL, Butterfield SL, Diamond DL, Young JC, Raves JJ, Cottington E, et al. Acute mortality associated with injuries to the pelvic ring: the role of early patient mobilization and external fixation. J Trauma. 1993;35(5):671-5.

6. Wali MA. Internal iliac artery injury in a fractured pelvis. Ann Thorac Cardiovasc Surg. 2003;9(5):337-9.

7. Mahendran B, Hynes N, Akhtar Y, Jawad A, Tawfik S, Courtney D, et al. Endovascular management of traumatic iliac vessel disruption-Report of two cases. EJVES Extra. 2005;9(6): 131-4.

8. Saad NE, Saad WE, Davies MG, Waldman DL, Fultz PJ, Rubens DJ. Pseudoaneurysms and the role of minimally invasive techniques in their management. Radiographics. 2005;25(Suppl 1):S173-89.

9. Astarci P, Alexandrescu V, Hammer F, Elkhoury G, Noirhomme P, Rubay J, et al. Late Presentation of Bleeding from a Traumatic Obturator Artery Aneurysm, Successfully Treated by Endovascular Means EJVES Extra. 2005;10(3):77-80.

10. Roblin P, Alexiou T, Sabharwal T, Reidy J, Ross DA. Successful stent-graft last angiography showed that it had undergone thrombosis, together with the small collateral branch, without extravasation of contrast medium on the angiography. In addition, while the patient was under observation, he remained stable, without any fall in hemoglobin and without changes to the D-dimers. The hematic infiltrate in the dorsal region went on gradually decreasing.

The natural history of pseudoaneurysms is unknown. However, some pseudoaneurysms, like those that affect the femoral artery and the superior thyroid artery, may undergo spontaneous thrombosis. Such occurrences are related to the size of the aneurysm, the neck length of the pseudoaneurysm and the patient's state of anticoagulation ${ }^{(12)}$.

To our knowledge, this is the first report of spontaneous thrombosis of a pseudoaneurysm in a branch of the iliac artery, in a patient with a pelvic fracture.

Thus, it is only in stable patients that clinical monitoring to verify the evolution of the disease prior to treatment can be relied on.

placement for the treatment of a superior gluteal artery pseudoaneurysm in a patient following complex pelvic surgery. Br J Radiol. 2007;80(949):e7-10.

11. Melissano G, Venturini M, Baccellieri D, Calliari F, Del Maschio A, Chiesa R. Distal embolization and proximal stent-graft deployment: a dual approach to endovascular treatment of ruptured superior gluteal artery aneurysm. Tex Heart Inst J. 2008;35(1):50-3.

12. Lee D, Legiehn GM, Munk PL. Pseudoaneurysm of the superior gluteal artery following polytrauma. Skeletal Radiol. 2007;36(9):875-8

13. Barlas A, Aribal E, Yegen C. Pseudoaneurysm of the left gluteal artery after a pelvic fracture sustained during the Marmara earthquake: report of a case. Surg Today. 2001;31(8):751-3.

14. El Khoury M, Mesurolle B, Kao E, Mujoomdar A, Tremblay F. Spontaneous thrombosis of pseudoaneurysm of the breast related to core biopsy. AJR Am J Roentgenol. 2007;189(6):W309-11.

15. Silva RM, Cury RC, Donich R, Batista L, Sá F, Loureiro F, et al. Tratamento endovascular de um pseudo-aneurisma de um ramo de artéria ilíaca interna esquerda. Rev Angiol Cirurg Vasc. 2007;(3)-13-4.

16. Esra Özkavukcu, Erdem Çaylı, Cemil Yağcı, İlhan Erden. Ruptured iliac aneurysm presenting as lumbosacral plexopathy. Diagn Interv Radiol. 2008;14(1):268.

17. Singh $D$,. Pinjala RK. Ruptured pseudoaneurysm of the gluteal artery . Intern $\mathrm{J}$ Surg. 2006;8(1). Disponível em: http://www.ispub.com/journal/the_internet_journal_of_surgery/volume_8_number_1/article/ruptured_pseudoaneurysm_of_the gluteal_artery.html. 